\title{
Ethical Issues in Interoperability of Electronic Healthcare Systems
}

\author{
Iroju Olaronke Ganiat \\ Computer Science Department, \\ Adeyemi College of Education, \\ Ondo, Ondo State, Nigeria
}

\author{
Olaleke Janet Olusola \\ Computer Science Department, \\ Adeyemi College of Education, \\ Ondo, Ondo State, Nigeria
}

\begin{abstract}
The advent of Information and Communication Technology (ICT) in the past decades has led to the wide adoption of electronic healthcare systems in healthcare. This has in actual fact led to the improvement in the quality of healthcare by enhancing the collection, storage, retrieval and access to health information. In addition, the use of ICT in healthcare provides more efficient healthcare services by reducing medical errors and costs thereby increasing patients' safety and satisfaction. Moreover, patients' information can be located in more than one electronic healthcare system. This is because patients may have more than one healthcare provider or they may move from one location to another. Hence, it becomes pertinent for patients' information to be available in all electronic healthcare systems at all points of care. Thus, the seamless exchange of meaningful information amongst healthcare providers and patients at the point of care becomes very crucial. This is because the diagnoses and treatment of patients depends on the timely access to accurate patients' information such as the medical history, laboratory reports as well as radiology report. However, several ethical challenges such as data privacy, confidentiality, control of access to patients' information, the commercialization of de-identified patients' information and ownership of patients' information which are associated with the interoperability of electronic healthcare systems still remain unresolved. Hence, the acceptance and adoption of electronic healthcare systems for information exchange and use is discouraged and hindered. Consequently, the healthcare system is associated with high error rates and low quality healthcare services. Thus, ethical issues need to be addressed in the context of interoperability of electronic healthcare systems. Based on this background, this paper appraises ethical issues associated with the interoperability of healthcare systems and how they can be addressed.
\end{abstract}

\section{General Terms}

Ethical issues, interoperability, healthcare systems

\section{Keywords}

Electronic healthcare systems, ethics, interoperability, Information and Communication Technology

\section{INTRODUCTION}

The ubiquitous nature of Information and Communication Technology (ICT) has led to a dramatic transformation in the healthcare system. Hence, the mode of communication, data collection/ acquisition, storage, processing and retrieval in the healthcare system is currently undergoing a rapid improvement. For instance, diverse ICT tools in the healthcare system involve devices that are usually worn or implanted in patients' bodies, as well as devices that are installed in patients' homes and their workplaces. These devices are basically used by healthcare practitioners to remotely monitor patients' information such as body temperature, heart rate, blood pressure, blood and urine levels, breathing rate and volume as well as other physiological conditions. ICT also has the potential of providing timely access to information that can be used to diagnose patients' health problems, thereby improving the quality and efficiency of healthcare services as well as reducing healthcare cost [1]. In addition, ICT in healthcare improves patients care by providing computerbased diagnostic devices such as the Computed Tomography (CT) scan, and Magnetic Resonance Imaging (MRI). Nevertheless, patients' information is typically managed by more than one healthcare provider such as nurses, physicians, laboratory technologists and radiologists. This is because patients can relocate from one geographical location to another or they can be referred from one healthcare institution to another. As a result, patients information are usually scattered across diverse proprietary systems. This leads to fragmented and heterogeneous data resources and services which contribute to the emergence of islands of information [2]. However, for healthcare providers within or across diverse healthcare institutions to have access to a patient's detailed and complete health history in a consistent, reliable and transparent manner, the concept of interoperability becomes imperative [1]. In addition, interoperability is necessary in healthcare because there is also no universally accepted format for data presentation.

According to the Institute of Electrical and Electronics Engineers Standard Computer Dictionary [3], interoperability is defined as the ability of two or more systems to exchange information and use the information that has been exchanged. Hence, interoperability facilitates the interactions amongst healthcare providers. For instance, an imaging procedure for a patient could be ordered by a physician in an external radiology center. Interoperability will enable the radiologist to have access to relevant clinical information of the patient from the physician database irrespective of his geographical location. Hence, Interoperability increases quality of care by facilitating seamless communication amongst healthcare providers. This facilitates the instant exchange of information amongst doctors, healthcare providers, laboratory, pharmacies, and clinics. Thus, interoperability increases the quality of patients care. However, ethical issues such as the protection of patients' data, patients' confidentiality and consent, data privacy, data integrity, control of access to patients' information, sale of de-identified patients' information, ownership of patients' information as well as the appropriate use of health information systems for data 
interoperability are key challenges facing the interoperability of electronic healthcare systems. Consequently, legal actions can be taken against healthcare practitioners who use electronic healthcare systems for healthcare delivery. Hence, the acceptance and adoption of electronic healthcare systems for information exchange and use is discouraged and hindered. Consequently, the healthcare system is associated with high error rates and low quality healthcare services. Based on this background, this paper identifies, analyses and discusses ethical issues associated with the interoperability of healthcare systems and how they can be addressed.

\section{ELECTRONIC HEALTHCARE SYSTEMS}

Electronic healthcare systems are ICT based tools that are used to store, access, retrieve, process, manage as well as control health information. They are also used to deliver quality healthcare services to patients. The different forms of electronic healthcare systems include the following:

\subsection{Electronic Health Record (EHR)}

This is a legal longitudinal health record of an individual in a digital format held in diverse media in the form of text, images, audio and video. EHR is usually composed of patient demographics, progress notes, medications, vital signs, medical history, immunizations, laboratory data, radiology reports amongst others. According to Winkelstein [4], EHRs represent more than a simple computerization of the traditional paper chart because they provide the ability to manage health information using modern information techniques that are impossible to apply to paper record keeping. However, EHRs are designed for easy access to health information by healthcare professionals that are involved in the patients' care [5]. Hence, EHR is designed to facilitate interoperability.

\subsection{Electronic Patient Record (EPR)}

This is a subset of the EHR. EPR however does not contain all the information of the patients but it is only limited to the patients information in one or more specialties such as dental medicine, surgery or intensive care unit.

\subsection{Electronic Medical Record (EMR)}

An Electronic Medical Record (EMR) according to World Health Organization [6] is a real-time patient health record with access to evidence-based decision support tools that can be used to aid clinicians in decision-making. The EMR can also be used for billing, quality management, outcome reporting, public health, disease surveillance and reporting. However, the information in an EMR is not easily shared by other healthcare providers [7].

\subsection{Personal Health Record (PHR)}

The PHR according to World Health Organization [6] is a universally accessible and comprehensible tool for managing relevant health information, promoting health maintenance and assisting with chronic disease management via an interactive, common data set of electronic health information and e-health tools. The PHR is usually created by patients with the intention of sharing their health data with their healthcare providers [7]. The PHR however differs from an EHR because it is not a legal record unless so defined [6].

\subsection{Patient-Carried Record (PCR)}

These are information that a patient carries on a token or card.
2.6 Mobile Health (mHealth or m-Health)

Mobile Health involves the delivering of health services and information via mobile technologies such as mobile phones and Personal Digital Assistants (PDAs).

\subsection{Telemedicine}

Telemedicine is the use of ICTs to provide and support healthcare services at a distance. Telemedicine involves the use of technologies such as video conferencing which allows physicians to interact with distant patients in real time [8].

\section{INTEROPERABILITY OF ELECTRONIC HEALTHCARE SYSTEMS}

In a general sense, interoperability is defined as the ability of two or more systems to exchange information, understand the exchanged information and use the information meaningfully for the purpose in which it was intended for in a meaningful and in a secured way. In the context of healthcare, interoperability can be viewed as the ability of healthcare systems to exchange information in a seamless way, use the information that has been exchanged meaningfully and also possess the ability to work together within and across organizational boundaries with the aim of providing improved healthcare services for individuals, communities, nations and the world at large. The use of information meaningfully in the definition above refers to the ability of electronic healthcare systems to improve the quality, safety, efficiency, and reduce health disparities; engage patients and family in care process, improve care coordination, population and public health, as well as maintain privacy and security of patient health information [7]. Thus, the concept of interoperability in healthcare can simply be described as the ability of System X to represent health information unambiguously as well as transmit the information (such as medications, drug names, diagnoses, allergies, immunizations, vital signs and chronic conditions) to System Y. Furthermore, System Y must be able to understand the meaning of the transmitted information, and use it for the purpose that is was intended for by System X. According to Siemens [9], interoperability of electronic healthcare system can be classified into four. These include syntactic interoperability, semantic interoperability, infrastructure interoperability and process interoperability. Syntactic interoperability has to do with the seamless exchange of health information amongst healthcare systems with the purpose of reducing the effects of distance in healthcare. Semantic interoperability refers to the capability of two or more electronic healthcare systems to understand and interpret the meaning of the information received accurately. Essentially, the electronic healthcare system and not just the healthcare practitioners must be able to understand the meaning of the exchanged information. Infrastructure interoperability refers to the ability of two or more medical devices to seamlessly work together by facilitating the electronic flow of information across the devices while process interoperability ensures seamless communication between different healthcare systems by developing a shared understanding of their process artifacts [10]. All levels on interoperability are inter-related.

\subsection{Benefits of Interoperability in}

\section{Healthcare}

The following are some of the benefits of interoperability in healthcare: 


\subsubsection{Enhanced Communication}

Patients usually consult different healthcare providers for care. Consequently, patients' medical records are usually scattered across several healthcare organizations. Hence, interoperability facilitates the collaboration, interaction and the instant exchange of information among healthcare providers irrespective of their geographical location during patients care. Hence, interoperability allows healthcare providers to have access to the complete medical history of a patient at the point of care regardless of the location of either the patient or the physician [11].

\subsubsection{Reuse of Information}

Interoperability facilitates the reuse of information instead of reentering the information each time a patient needs care.

\subsubsection{Easy Comprehension of Medical Terms}

Interoperability facilitates the easy comprehension of medical terms as data is transmitted from one system to another, while ensuring that the meaning of the information is not altered. Thus, interoperability ensures that the right meanings of medical terminologies are delivered to the interoperating systems.

\subsubsection{Reduction of Medical Errors}

More than one million patients are injured each year as a result of broken health care processes [12]. However, interoperability facilitates the integration of information from autonomously developed applications across healthcare systems. Interoperability therefore reduces medical errors by ensuring that the communicating systems understand the structure and content of the integrated data [1].

In spite of the numerous benefits of interoperability of electronic healthcare systems, most electronic healthcare systems are however not totally interoperable majorly because of the ethical issues in healthcare such as security of patients' information, privacy, consent, confidentiality, ownership of patients' information, de-identification of patients' information amongst others. Hence, less than $25 \%$ of the hospitals in the United States of America deploy electronic healthcare systems. Thus, with the vast amount of information available in healthcare, the challenge is to ensure that ethical principles are adequately addressed in order to foster interoperability of electronic healthcare systems.

\section{ETHICAL PRINCIPLES IN INTEROPERABILITY OF ELECTRONIC HEALTHCARE SYSTEMS}

Ethics is pluralistic [13]. According to Gammel [14], the term 'ethics' is derived from the Greek word 'ethos' which means customs, habits and morals of a people. There are various definitions for ethics. Ethics according to Williams [13], is the study of morality, a careful and systematic reflection on and analysis of moral decisions and behaviour, whether past, present or future. Ethics is a philosophical discipline which is concerned with the morality of human behaviour, with right and wrong [15]. Ethics also constitute the principles of right behaviour which guides the actions of individuals and groups. It investigates the values and virtues that are paramount to a society [16]. Ethics is also concerned with justice, rights, respect of human dignity, autonomy of the individual and respect for the community [17]. Hence, ethics in the interoperability of healthcare systems refers to practice, attitudes, values, principles and codes that strictly guide healthcare professionals and patients during the exchange and use of health information in an unambiguous way.
Interoperability in healthcare is inconceivable without the use of electronic healthcare systems. However, the use of ICT in healthcare raises significant ethical issues such as ownership of health information, benefits and risks of deploying ICT for patients care and how ICT has affected physician-patient relationship amongst others [18]. Thus, ethics are central to health information exchange as well as the care of patients [19].

The four key bioethical principles developed by Beauchamp and Childress [20] are hereby discussed in this section in relation to the interoperability of electronic healthcare systems. This is because Modern bioethics and health informatics are closely connected [4]

\subsection{Autonomy}

Autonomy is the right of an individual to determine his or her own healthcare [4]. Hence, patients must have a right to their health information despite that patients information are created and managed by healthcare practitioners. Thus, patients must be fully aware before their health information is exchanged amongst diverse healthcare providers. Patients should also be given the opportunity to give full consent of who to view their health information. Otherwise, patients' trust and confidence for their healthcare providers may be threatened, thus, preventing the patients from disclosing sensitive information required for diagnosis and treatment [21]. Hence, patient's information must not be divulged or revealed to anyone who is not involved in the care of the patient. However, there are exceptional cases where patients' information is divulged without the patients' consents. For example, the information about an individual with a deadly disease should be made known to the general public, such as the recent outbreak of the Ebola disease in West Africa in the year 2014. In addition, Almacen [7] is of the view that patients should not be given complete autonomy of their health information, because this will encourage the patients to modify or delete the information they do not want to share. Hence, an incomplete picture of the patient's true health condition may be presented during care and this could have life-threatening consequences. However, questions such as who owns the patients' information is still a cause for debate in healthcare.

\subsection{Beneficence}

According to Mercuri [22], beneficence simply means to do good. Beneficence supports healthcare providers to share information about a patient during care and most especially during emergencies in order to promote the general well-being of the patient. Hence, beneficence ensures that the information shared amongst healthcare providers is used in the best interests of the patients.

\subsection{Non-Malfeasance}

Non-malfeasance or primum non nocere in Latin means to do no harm [7]. Harm in this parlance refers to anything which worsens the condition of the patients such as the introduction of pain, discomfort, suffering, disability or disfigurement, and death to a patient. Hence, non- malfeasance is a process of preventing harm to patients as long as it is within the ability of healthcare providers and without undue harm to themselves. Non- malfeasance therefore ensures that the information shared during interoperability is made available to authorized individuals when needed for diagnosis, treatment or research and the information shared is used to improve the health status of the patient. 


\subsection{Justice}

Justice could simply mean fairness or equality. Justice is observed in the interoperability of health information if interoperating electronic healthcare systems are used to provide equal and prompt healthcare care to everyone as well as also ensuring data availability, accuracy, and security [7]. The principle of justice is beginning to emerge as a significant factor that bridges the digital divide that exists between the "haves" and "have-nots". The principle of justice also facilitates the respect of privacy, confidentiality and security of healthcare information during information exchange and use [7].

\section{RELEVANCE OF ETHICAL PRINCIPLES TO THE INTEROPERABILITY OF ELECTRONIC HEALTHCARE SYSTEMS}

The following are the significance of ethical principles to the interoperability of electronic healthcare systems.

\subsection{Enhanced Patients Confidentiality}

By observing ethical principles in the interoperability of electronic healthcare systems, respect for persons, informed consent and protection of confidentiality of patients' information will be enhanced. Ethical principles in the interoperability of healthcare systems will facilitate respect for individuals by preserving their privacy which is often greatly compromised. Hence, information about an individual will not be made known to others without his or her consent unless authorized by the individual concerned.

\subsection{Trust}

Ethics enhances trust between patients and physicians and facilitates the relationship between them. In the course of care, patients give their personal information to healthcare practitioners such as nurses and physicians. In addition, patients' information can also be obtained through tests. This information might have to be shared amongst diverse healthcare practitioners at the point of care. However, with ethical principles, patients must be able to trust their caregivers not to disclose/divulge their information unless stated by law. Otherwise, patients may refuse to give personal information which can hinder effective diagnosis as well as treatment.

\subsection{Autonomy}

Ethical principles also grant patients the opportunity to have a right to their health information as well as the right to determine their own health care. By observing ethical principles, patients will have a level of control over their health information during information exchange amongst healthcare practitioners. Hence, the unauthorized disclosure of sensitive information such as sexual abuse, drug abuse and health conditions such as Human Immuno-Deficiency Virus (HIV) will be prevented.

\subsection{Fair Distribution of Healthcare \\ Resources}

Ethics ensures fair distribution of healthcare resources amongst patients without any form of discrimination. Ethical principles also ensure that healthcare practitioners advocate for more resources from government or other healthcare agencies in order to meet the healthcare needs of patients in cases where the resources are limited.

\subsection{Ethical Principles Guarantee Patients Safety}

Electronic healthcare systems are prone to a number of security violations which include unauthorized view of patients' information, unauthorized modification to patients' information, unauthorized destruction of patients' data as well as information theft. Hence, the safety of patients' is in jeopardy. However, by observing ethical principles in the interoperability of healthcare systems, patients' safety is guaranteed. This will ensure that only authorized individuals have access to patients' information.

\section{CHALLENGES OF ETHICAL PRINCIPLES IN THE INTEROPERABILITY OF ELECTRONIC HEALTHCARE SYSTEMS}

The following are some of the barriers to ethical principles in the interoperability of healthcare systems

\subsection{Security}

Patients' information is usually scattered across several autonomous, heterogeneous and proprietary healthcare systems which are usually shared amongst diverse healthcare professionals. In addition, the digitization of patient records has led to the increased access of heath information via electronic healthcare systems most especially mobile devices (such as smartphones). Hence, health information is prone to security challenges such as the unauthorized access to health information by malicious persons, medical fraud, unauthorized modification of patients' information, data hacking, unauthorized destruction of patients' data as well as eavesdropping of patient information over a network [23]. In 2011, the Ponemon Institute [24] reported that security breaches in healthcare in the United States of America (USA) rose to $32 \%$ and this led to a loss of about $\$ 6.5$ billion in the healthcare industry. Hence, patients are usually unwilling to share sensitive information through electronic healthcare systems.

\subsection{Privacy and Confidentiality}

According to Winkelstein [4], privacy involves the ability of a patient to control the information about him or herself. Privacy in healthcare can also be viewed as the ability to protect patients' information from unauthorized access and disclosure [25]. Confidentiality on the other hand is the commitment of another person or organization to control information about the patient [4]. Hence, confidentiality refers to the ability of healthcare practitioners to make data available to only authorized persons or processes. However, healthcare systems are vulnerable to privacy and confidentiality challenges because of the nature of information it acquires, stores and transmits. Examples of this information include the social security number of the patient, insurance identification, payment information, and medical provider information [26].

\subsection{Ownership of Patients' Information}

The issue of who owns the patient's information has been contentious within the healthcare system as this varies from country to country. For instance, Odom-Wesley [27] is of the view that the healthcare professionals own the physical medical record since it is a business record and property, while the patients' own the information in the record. However, Almacen [7] argues that healthcare providers may claim ownership over a patient's EHR. Almacen [7] also emphasized that patients should not be given complete control over their information as they may alter or delete parts or all 
of the records that they do not want to share. Hence, an incomplete and inaccurate picture of the patient's true health condition is presented at the point of care. Thus, the question of who owns the patients information is still debatable in the context of the healthcare system.

\subsection{Commercialization of De-identified Patients' Information}

In simple terms, de-identification can be defined as the process of preventing a patient's identity from being connected with his information. Patient's information is usually composed of the demographic information (such as the name of the patient, the gender, date of birth, address, zip code), payment information about healthcare provision and social security number. Healthcare EHR vendors such as Cerner and All scripts which was formerly known as Eclipsys sold de-identified copies of their patients databases to pharmaceutical companies, medical device makers, and health services researchers [28]. Moreover, patients can easily be identified by a combination of their information such as name, address, date of birth, patient record number, diseases associated with the individual, drug regime, zip code as well as gender. Hence, the risk of re-identifying the patient is pronounced and thus the breach of confidentiality and privacy is increased.

\section{A COMPARATIVE ANALYSIS OF EXISTING STUDIES ON ETHICAL ISSUES IN ELECTRONIC HEALTHCARE SYSTEMS}

A lot of studies have been conducted on ethical issues in electronic healthcare systems. Hence, this section appraises and compares the diverse views of different authors on ethical issues in electronic healthcare systems. According to Beauchamp and Childress [20], the four basic ethical principles that are pertinent to electronic healthcare systems include autonomy, beneficence, non-malfeasance and justice. Snydey and Gauthier [29] viewed the principles of dignity and veracity as basic ethical principles in electronic healthcare systems in addition to the four basic ethical principles developed by Beauchamp and Childress [20]. According to [29], the principle of dignity is based on the idea that all persons have a right to be treated with respect and dignity. Respect for dignity according to [29] includes respect for their emotions, relationships, reasonable goals, privacy, and bodily integrity. The principle of veracity is the right of individuals to be provided with complete, accurate and truthful information about their medical conditions at the point of diagnosis or treatment. On the other hand, Reidl [30] emphasized that the basic ethical principles of electronic healthcare systems include the principles of transparency, standardization, work ethics, privacy and confidentiality as well as the principle of equitable allocation of healthcare resources. The principle of transparency according to Reidl [30] is the process of providing the users of the electronic healthcare system with a valid and simple model of the system. This implies that the users must have an adequate knowledge of the effects of the system on their activities. The principle of standardization ensures that the standards used by the electronic healthcare systems do not disrupt the local work practices of the healthcare practitioners, while the principle of work ethics ensures that the ICT facilities used in the healthcare system augment the activities of the healthcare practitioners. The principle of privacy and confidentiality according to Reidl [30] ensures that the electronic healthcare system provides surveillance to its users. This ensures that unauthorized access to electronic health information is prevented. The principle of equitable allocation of healthcare resources is similar to the principle of justice developed by Beauchamp and Childress [20]. This principle ensures that healthcare facilities are available to all individuals at the point of care. In addition, the ethical principles of electronic healthcare systems according to the International Medical Informatics Association (IMIA) code of conduct include the principles of information-privacy and disposition, openness, security, access, legitimate infringement and the principle of accountability. The principle of information-privacy and disposition emphasizes that all individual must have a fundamental right to control the collection, storage, access, use, communication, manipulation and disposition of data about themselves. The principle of openness ensures that the collection, storage, access, use, communication, manipulation and disposition of personal data are released in an appropriate and timely fashion to the subject of those data [31]. The principle of security guarantees that health information are protected against loss, degradation, unauthorized access and destruction. The principle of access ensures that an individual has the right to access his information and the right to modify his information with respect to accuracy, completeness and relevance [31]. The principle of legitimate infringement according to [31] ensures that the fundamental right of control over the collection, storage, access, use, manipulation, communication and disposition of personal data is conditioned only by the legitimate, appropriate and relevant data-needs of a free, responsible and democratic society while the principle of accountability ensures that any infringement of the privacy right of an individual is justified in an appropriate and in a timely manner. Table 1 shows a summary of the comparative analysis of the existing studies on ethical issues in electronic healthcare systems.

Table 1. A comparative analysis of the existing studies on ethical issues in electronic healthcare systems

\begin{tabular}{|c|l|l|}
\hline S/N & \multicolumn{1}{|c|}{ Authors } & \multicolumn{1}{c|}{ Ethical Principles Developed } \\
\hline 1 & $\begin{array}{l}\text { Beauchamp and } \\
\text { Childress }\end{array}$ & $\begin{array}{l}\text { autonomy, beneficence, non- } \\
\text { malfeasance and justice }\end{array}$ \\
\hline 2 & $\begin{array}{l}\text { Snydey and } \\
\text { Gauthier }\end{array}$ & $\begin{array}{l}\text { autonomy, beneficence, non- } \\
\text { malfeasance, justice, dignity and } \\
\text { veracity }\end{array}$ \\
\hline 3 & Reidl & $\begin{array}{l}\text { transparency, standardization, work } \\
\text { ethics, privacy and equitable } \\
\text { allocation of healthcare resources }\end{array}$ \\
\hline 4 & $\begin{array}{l}\text { IMIA code of } \\
\text { conduct }\end{array}$ & $\begin{array}{l}\text { information-privacy and disposition, } \\
\text { openness, security, access, } \\
\text { legitimate infringement and the } \\
\text { principle of accountability }\end{array}$ \\
\hline
\end{tabular}

\section{RECOMMENDATIONS}

The following are recommendations on how ethical challenges can be addressed in the context of interoperability of electronic healthcare systems.

\subsection{Anonymisation of De-Identified Patients' Information}

In cases where de-identified patients information has to be commercialized for research and other purposes, it is recommended that the patients' information be anonymised 
by removing information that can make the patients to be easily identified.

\subsection{Informed Consent}

Patients should be duly informed before their personal information is shared amongst diverse healthcare providers unless otherwise stated by law such as patients information needed for the investigation of a serious crime such as in the case of murder. This will enhance physician-patient relationship and build trust amongst physicians and patients.

\subsection{Adequate Security Measures}

Adequate security measures such as the use of audit trail systems should be put in place to constantly monitor all activities that take place during information exchange and use in electronic healthcare systems such as who accesses the data, which data was accessed and when the data was accessed.

\section{CONCLUSION}

The importance of interoperability of electronic healthcare systems cannot be underestimated. This is because interoperability enhances communication amongst diverse healthcare practitioners involved in the care of a patient, interoperability facilitates the reuse of healthcare information, it reduces medical errors as well as facilitates the comprehension of medical terms. However, interoperability is yet to be achieved within the context of healthcare systems majorly because of ethical challenges in healthcare such as security of patients' information, privacy, confidentiality, ownership of patients' information, sale of de-identified patients' information amongst others. In view of this, this paper views the four major ethical principles in healthcare with respect to the interoperability of electronic healthcare systems. These ethical principles include the principles of autonomy, beneficence, non-malfeasance and justice. This paper also examines the importance of ethical principles to the interoperability of electronic healthcare systems. The paper highlights autonomy, patients' safety, fair distribution of healthcare resources and trust as the benefits of ethical principles in the interoperability of electronic healthcare systems. This paper also analyzes the challenges facing ethical principles in the interoperability of electronic healthcare systems. These include security, privacy and confidentiality, ownership of patients' information as well as the sale of de-identified patients' records. The paper however recommends that the use of adequate security measures to monitor the activities taking place within interoperating healthcare system, the anonymisation of de-identified patients' information as well as getting the consent of the patients before their information is shared are some of the ways in which the challenges facing the ethical principles of interoperability of electronic healthcare systems can be addressed.

\section{REFERENCES}

[1] Iroju, O., Soriyan, A., Gambo, I. and Olaleke, J. 2013. Interoperability in healthcare: benefits, challenges and resolutions. International Journal of Innovation and Applied Studies, 3 (Mar, 2013), 262-270.

[2] Ikono, R., Soriyan, A., Iroju, O., and Gambo, I. 2014. A survey of the barriers to interoperability of Nigeria healthcare systems. In Proceedings of the 9th Health Informatics in Africa Conference.
[3] Institute of Electrical and Electronics Engineers Standard Computer Dictionary, 1999. A Compilation of IEEE Standard Computer Glossaries, Institute of Electrical and Electronics Engineers, USA.

[4] Winkelstein, P.S. 2009. Ethical and social challenges of Electronic health information. Medical Informatics.139159.

[5] Garrett, P., and Seidman, J. 2011. EMR vs. EHR - What is the difference? , 4 (Jan, 2011).

[6] World Health Organization. 2012. Management of patient information trends and challenges in member states. Global Observatory for eHealth series.

[7] Almacen, M. 2013. EHR interoperability: legal, ethical, and social challenges. Northwestern University Evanston, Illinois.

[8] Burney, S. M. A., Mahmood, N. and Abbas Z. 2010. Information and Communication Technology in Healthcare. International Journal of Computer Applications. (Jul, 2010), 27-32.

[9] Siemens. 2011. Interoperability in Healthcare. A White Paper on the Interoperability of Healthcare Systems. (July, 2011).

[10] Khan, W. A., Hussain, M., Latif, K., Afzal, M., Ahmad, F. and Lee, S. 2013. Process interoperability in healthcare systems. Springer-Verlag Wien, 1-26.

[11] Iroju, O., Ikono, R., Gambo, I., and Soriyan,A. 2014. Impacts of usability on the interoperability of electronic healthcare systems. International Journal of Innovation and Applied Studies. 2(Sep, 2014), 827-832.

[12] Pan, E., Johnston, D. Walker, J., Adler-Milstein, J., Bates D. W. and Middleton, B. 2004. The value of healthcare information exchange and interoperability. Center for Information Technology Leadership, Wellesley.

[13] Williams, J.R. 2009. Medical ethics manual. World Medical Association.

[14] Gammel S. 2005. Ethics and morality. Technical University Darmstadt.

[15] Itulua-Abumere, F. 2012. Ethical Issues in Health and Social Care Profession. OSR Journal of Humanities and Social Science (JHSS). 14-18

[16] Frankena, W. 1973. Ethics. Prentice Hall.

[17] Gabr, M. 1997. Health ethics, equity and human dignity. Faculty of Medicine, Cairo University, Egypt.

[18] Marckmann, G. and Goodman, K. W. 2006. Introduction: Ethics of Information Technology in Health Care. International Review of Information Ethics.

[19] Limentani, A.E. 2009. The role of ethical principles in health care and the implications for ethical codes. Journal of Medical Ethics. 11 (Feb, 2009), 394-398.

[20] Beauchamp, T.L. and Childress, J.F. (1994). Principles of biomedical ethics. Oxford University Press, New York.

[21] Layman, E. J. 2008. Ethical issues and the electronic health record. Retrieved from: 
http://www.ncbi.nlm.nih.gov/pubmed/18475119?report= abstract.

[22] Mercuri, J. J. 2010. The ethics of electronic health $\begin{array}{lll}\text { records. } & \text { Retrieved } & \text { from } \\ \text { http://www.clinicalcorrelations.org/?p=2211 }\end{array}$

[23] Iroju, O. and Ikono, R. 2013. A security based framework for interoperability of healthcare systems. International Journal of Applied Information Systems. 23-31.

[24] Ponemon Institute, 2010. Preventative care, proactive response. Mission College Boulevard Santa Clara, USA

[25] Cooper ,T. and Collman, J. 2006. Managing information security and privacy in healthcare data mining state of the art. Department of Ophthalmology, Stanford University Medical School, Palo Alto, California.

[26] Keckley,P.H. 2010. Privacy and security in health care: a fresh look. Deloitte Center for Health Solutions, USA.
[27] Odom-Wesley, B., Brown, D., and Meyers, C.L. 2009. Documentation for medical records. American Health Information Management Association, Chicago.

[28] Keckley,P.H. 2010. Privacy and security in health care: a fresh look. Deloitte Center for Health Solutions, USA.

[29] Snydey, J.E. and Gauthier, C.C. 2008. The underlying principles of ethical patient care. Evidence-Based Medical Ethics:Cases for Practice Based Learning. Springer.

[30] Reidl, C. 2005. Examining the ethical issues of it in healthcare. Institute for Technology Assessment and Design, Vienna University of Healthcare.

[31] International Medical Informatics Association 2002. The IMIA code of ethics for health information professionals. International Medical Informatics Association 